\title{
Agent-Based Distributed Manufacturing Process Planning and Scheduling: A State-of-the-Art Survey
}

\author{
Weiming Shen, Senior Member, IEEE, Lihui Wang, and Qi Hao
}

\begin{abstract}
Manufacturing process planning is the process of selecting and sequencing manufacturing processes such that they achieve one or more goals and satisfy a set of domain constraints. Manufacturing scheduling is the process of selecting a process plan and assigning manufacturing resources for specific time periods to the set of manufacturing processes in the plan. It is, in fact, an optimization process by which limited manufacturing resources are allocated over time among parallel and sequential activities. Manufacturing process planning and scheduling are usually considered to be two separate and distinct phases. Traditional optimization approaches to these problems do not consider the constraints of both domains simultaneously and result in suboptimal solutions. Without considering real-time machine workloads and shop floor dynamics, process plans may become suboptimal or even invalid at the time of execution. Therefore, there is a need for the integration of manufacturing process-planning and scheduling systems for generating more realistic and effective plans. After describing the complexity of the manufacturing process-planning and scheduling problems, this paper reviews the research literature on manufacturing process planning, scheduling as well as their integration, particularly on agent-based approaches to these difficult problems. Major issues in these research areas are discussed, and research opportunities and challenges are identified.
\end{abstract}

Index Terms-Agents, distributed manufacturing systems, manufacturing scheduling, multiagent systems, process planning.

\section{INTRODUCTION}

$\mathbf{M}$ ANUFACTURING process planning and scheduling are usually considered to be two separate activities in manufacturing. Manufacturing process planning determines how a product will be manufactured. It is the process of selecting and sequencing manufacturing processes and parameters so that they achieve one or more goals (e.g., lower cost, shorter processing time, etc.) and satisfy a set of domain constraints. Manufacturing scheduling, on the other hand, is the process of assigning manufacturing resources over time to the set of manufacturing processes in the process plan. It determines the most appropriate time to execute each operation, taking into account the temporal relationships between manufacturing processes and the capacity limitations of the shared manufacturing resources. The assignments also affect the optimality of a schedule with respect to criteria such as cost, tardiness, or throughput. In summary, scheduling is an optimization process where limited resources are allocated over time among both parallel and sequential activities [136]. Such an optimization process is becoming increas-

Manuscript received December 5, 2003; revised February 3, 2005 and June 29, 2005. This paper was recommended by Associate Editor V. Marik.

The authors are with Integrated Manufacturing Technologies Institute, National Research Council Canada, London, ON, Canada (e-mail: weiming.shen@ nrc.gc.ca; lihui.wang@nrc.gc.ca; qi.hao@nrc.gc.ca).

Digital Object Identifier 10.1109/TSMCC.2006.874022 ingly important for manufacturing enterprises to increase their productivity and profitability through greater shop floor agility to survive in a globally competitive market [98].

This paper describes the complexity of manufacturing process-planning and scheduling problems (Section II), and reviews the research literature in manufacturing process planning (Section III), manufacturing scheduling (Section IV), and the integration of process planning and scheduling (Section V), particularly focusing on agent-based approaches in these areas. Major issues in these research areas are discussed (Section VI), research opportunities and challenges addressed (Section VII), and a brief conclusion stated (Section VIII).

The objective of this paper is not to provide an extensive survey of general manufacturing process-planning and scheduling systems, but to focus on the agent-based approaches and their applications in manufacturing process planning and scheduling. An earlier survey of multiagent systems for intelligent manufacturing systems, including agent-based manufacturing process planning, scheduling, and control, can be found in [92]. More discussions on the applications of agent technology to collaborative design and manufacturing can be found in [94].

\section{PRoblem Complexity}

The problem of manufacturing process planning and scheduling has been introduced in Section I. This section discusses the complexity of the problem and the difficulty in solving it.

The scheduling problem exists not only in manufacturing enterprises, but also in organizations like publishing houses, universities, hospitals, airports, and transportation companies. It is typically NP-hard, i.e., it is impossible to find an optimal solution without the use of an essentially enumerative algorithm, with computation time increasing exponentially with problem size. However, the manufacturing scheduling problem is one of the most difficult of all scheduling problems. More detailed discussions and analyses of scheduling problems can be found in [5], [29].

A well-known manufacturing scheduling problem is the classical job shop scheduling where a set of jobs and a set of machines are given. Each machine can handle at most one job at a time. Each job consists of a chain of operations, each of which needs to be processed during an uninterrupted time period of given length on a given machine. The purpose is to find the best schedule, i.e., an allocation of the operations to time intervals on the machines, that has the minimum total duration required to complete all jobs. The total number of possible solutions for a classical job shop scheduling problem with $n$ jobs and $m$ machines is $(n !)^{m}$ [5]. 
The problem becomes even more complex in the following situations.

1) When other manufacturing resources, such as operators and tools, are also considered during the scheduling process. For a classical job shop scheduling problem with $n$ jobs, $m$ machines, and $k$ operators, the total number of possible solutions could be $\left((n !)^{m}\right)^{k}$.

2) When both process planning and manufacturing scheduling are to be done at the same time. Traditional approaches that treat process planning and manufacturing scheduling separately can result in suboptimal solutions for the two phases. Integrating the two phases into one optimization problem, by considering the constraints of both domains simultaneously, can theoretically result in a global optimal solution, but it increases the solution space significantly.

3) When unforeseen dynamic situations are considered. In a job shop manufacturing environment, rarely do things go as expected. The system may be asked to include additional tasks that are not anticipated, or to adapt to changes to several tasks, or to neglect certain tasks. The resources available for performing tasks are subject to changes. Certain resources can become unavailable, and additional resources can be introduced. The beginning time and the processing time of a task are also subject to variations. A task can take more or less time than anticipated, and tasks can arrive early or late. Other uncertainties include power system failures, machine failures, operator absence, and unavailability of tools and materials. An optimal schedule, generated after considerable effort, may rapidly become unacceptable because of unforeseen dynamic situations on the shop floor and a new schedule may have to be generated. This kind of rescheduling problem is also called dynamic scheduling or real-time scheduling.

\section{ApProaches to ManUfacturing Process PlanNing}

\section{A. Traditional Approaches}

Traditionally, manufacturing process planning is a task that transforms design information into manufacturing processes and determines the sequence of operations [15]. Maintaining the consistency of process plans and keeps them optimized is a difficult task. Since 1965, when Nieble [74] reported the first computer-aided process planning (CAPP) system, numerous research efforts have been reported in this area.

Generally, CAPP approaches can be classified into two categories: variant and generative. The success of the variant approach depends on group technology and computerized database retrieval. When a new part enters a factory, a previous similar process plan is retrieved from the database and modified to suit the new part. This method is especially suitable for companies with few, and relatively fixed, product families and a large number of parts per family. Most of the earlier CAPP systems can be categorized under the variant approach [2]. The generative approach, on the other hand, can be used to automatically generate an optimal process plan according to the part's features and manufacturing requirements. Most of the generative systems in the literature are knowledge-based systems utilizing artificial intelligence techniques. They are oriented toward the needs of large companies, especially those producing products with large variety and small batch sizes. However, a truly generative process-planning system that can meet industrial needs and provide an appropriate generic framework, knowledge representation methods, and inference mechanisms has not been developed so far [134].

Various approaches to CAPP have been proposed in the literature [2], [25]. Research studies on process planning include object-oriented approaches [105], [132], GA-based approaches [70], [131], neural-network-based approaches [21], [69], Petri net-based approaches [53], feature recognition or feature-driven approaches [114], [119], and knowledge-based approaches [108], [118]. These approaches and their combinations have been applied to some specific problem domains, such as tool selection [24], [56], tool path planning [7], [45], machining parameters selection [3], [37], process sequencing [129], and setup planning [75], [125].

Recently, the research focus on process planning has shifted toward solving problems in distributed manufacturing environments. Tu et al. [115] introduced a method called incremental process planning (IPP) for one-of-a-kind production (OKP) in such environments. The IPP is used to extend or modify a primitive plan (a skeletal process plan) incrementally according to new features that are identified from a product design until no more new features can be found. A complete process plan generated by the IPP may include alternative processes.

\section{B. Agent-Based Approaches}

Apart from centralized AI approaches [e.g., genetic algorithms (GAs), neural networks, fuzzy logic, and expert systems], agent technology is emerging as a solution for distributed AI that has attracted a wide attention. Instead of being one large expert system, cooperative intelligent agents are being used in developing distributed CAPP systems. The agent-based approach is also being recognized as an effective way to realize adaptiveness and dynamism of process planning. The following are some examples of agent-based process-planning systems.

1) Shih and Srihari [99] proposed a distributed AI-based framework for process planning. Their approach decomposes the entire production control task into several subtasks, each of which is implemented by an intelligent agent. By working collaboratively, the agents can reach a solution for the problem.

2) CoCAPP [133], [134] was proposed to distribute complex process-planning activities to multiple specialized problem solvers and to coordinate them to solve complex problems. The CoCAPP attempts to satisfy five major requirements: autonomy, flexibility, interoperability, modularity, and scalability. It builds cooperation and coordination mechanisms into distributed agents using knowledge-based techniques. Each agent in the system deals with a relatively independent functional domain in process planning.

3) Zhang et al. [132] proposed an agent-based adaptive process-planning (AAPP) system on top of an 
object-oriented manufacturing resources modeling (OOMRM) framework. The OOMRM describes manufacturing resources' capability and capacity in an object-oriented manner, while the AAPP is implemented as an integrated process-planning platform. Instead of automating process-planning tasks completely, the AAPP system provides an interactive mode to enable experienced manufacturing engineers to make decisions at crucial points. Five agents are used in the AAPP to carry out part information classification, manufacturing resources mapping, process planning, human planning, and machining parameter retrieval. A contract net-based scheme is utilized as the coordination protocol between agents.

4) Sluga et al. [102] introduced a virtual work system (VWS) as the essential building block for in a distributed manufacturing environment. The VWS represents a manufacturing work system in the information space, and is structured as an autonomous agent. It is a constituent entity of an agent network in which dynamic clusters of cooperating agents are solving manufacturing tasks. The decision-making in process planning is based on a market mechanism consisting of bidding-negotiationcontracting phases. The VWS approach aims at enabling dynamic decision-making based on the actual state of the manufacturing environment.

5) CyberCut [103] is a research project that aims at developing a networked manufacturing service for rapid part design and fabrication on the Internet. A critical part of this service is an automated process-planning module that is capable of generating process plans to satisfy the desired geometries and specified requirements. Three types of agents are designed to facilitate CyberCut: primary process-planning agent, environmental planning agent, and burr minimization tool path planning agent [22]. The multiagent planning module incorporates conventional and specialized planning agents for environmental consideration and burr minimization. However, the interactions between agents are based on human decisions.

6) IDCPPS [14] was reported to be an integrated, distributed, and cooperative process-planning system. The processplanning tasks are broken into three levels, namely, initial planning, decision-making, and detail planning. The initial planning deals with the manufacturability evaluation of a design and the generation of alternative processing routes based on feature reasoning. The decision-making level takes place when the orders have been released for production on the shop floor. The result of this step is a ranked list of near-optimal alternative plans that considers the availability of shop floor resources. The detail planning is executed just before manufacturing begins. This step finishes the final selection of machines, tools, cutting parameters, and the calculation of machining cost and time. Different functional modules are grouped into different agents, including the three process-planning agents dealing with the above three-level planning, plus the task agents, resource agents, and coordination agents (CAD/Process coordination agent and Process/Production coordination agent).
However, the whole framework seems to have been designed at a high level. No practical systems were reported.

7) Similarly, Lim and Zhang [55] introduced an APPSS system, which is made up of a number of agents and functional modules. This system is mainly used for the dynamic reconfiguration and optimization of resource utilization in manufacturing shop floors by considering the real-time process-planning and scheduling issues.

8) Kornienko et al. [50] considered process planning as a typical constraint satisfaction problem to generate an optimized plan in a distributed way satisfying all restrictions in the presence of different disturbances. An agent plays different "roles" and has a primary algorithm (determined by interactive pattern) and a set of emergency states to handle local emergencies or global emergencies. In case an agent is in emergency state recognized by the activity guard agent, it could either resolve the emergency by itself or request a rescue agent to handle it.

In addition to the above systems, there are also other similar research efforts toward agent-based process planning [78], [110]. All these systems tend to solve the process-planning problem by cooperation and negotiation among intelligent agents. The agents making up the systems usually use the function decomposition approach as described in Section VI.

\section{APPROACHES TO MANUFACTURING SCHEDULING}

\section{A. Traditional Approaches}

Because of its highly combinatorial aspects (NP-complete), dynamic nature, and practical usefulness for industrial applications, the scheduling problem has been widely studied in the literature by various methods: heuristics, constraint propagation techniques, constraint satisfaction problem formalisms, Tabu search, simulated annealing, GAs, neural networks, fuzzy logic, etc. [136].

As direct methods are not available for complex scheduling problems, search methods are usually adopted to solve these problems. However, the simplest generate-and-test search strategy is not a reasonable approach for large complex problems. Many local search algorithms are more appropriate. These algorithms require a cost function, a neighborhood function, and an efficient method for exploring the neighborhood.

A variety of neighborhood search methods have been created including climbing, simulated annealing, etc. These methods offer heuristic refinements to the generate-and-test. Heuristic approaches try to replace the exhaustive search strategies with some sophisticated experience. With the aid of heuristics in searching strategies, good solutions (though possibly nonoptimal) to hard problems can be found within greatly reduced computation time.

The Petri Net approach and its variants, due to its graphical representation and mathematical analysis of the control logic of a manufacturing system, provide a powerful approach to model, control, and schedule an automated system, in both its information flows and its material flows. Colored timed object-oriented Petri Nets (CTOPN) [123] further incorporates 
structured, reusable, and easily maintainable control/decision knowledge that can be used in scheduling/dispatching.

Constraint satisfaction is another search procedure that operates in the space of constraint sets rather than in the solution set space [59], [60], [68].

The objective of multisite scheduling [86] is to support the scheduling activities of a global scheduler or schedulers in distributed production plants in a cooperative way. A schedule generated on a global level must be translated into detailed schedules as part of the local scheduling process. In the case of a disturbance, feedback between the local and global levels is essential. Global-level data are derived from aggregated local data, and are normally imprecise or estimated.

Several approaches take advantages of search strategies in which even cost-deteriorating neighbors are accepted. Simulated annealing uses an analogy with the physical process of annealing, in which a pure lattice structure of a solid is made by heating up the solid in a heat bath until it melts, then cooling it down slowly until it solidifies into a low-energy state. As designed, simulated annealing is a randomized neighborhood search algorithm and it has been successfully applied to solve many single-objective scheduling problems. Tabu search combines deterministic iterative improvements with the possibility of accepting cost-increasing solutions occasionallyto direct the search away from local minimum [32]. In GAs, learning occurs through a solution selection process. GAs discover superior solutions to global optimization problems adaptively (akin to the evolution of organisms in the natural world) by searching for small, local improvements rather than big jumps in a solution space. Fuzzy logic-based scheduling is used to support the scheduling activities in a multisite scheduling scenario [86]. In this system, a global scheduler or schedulers in distributed production plants work in a cooperative way, based on adequate modeling and processing of imprecise data. A robust prescription is created for the local scheduling systems.

All the traditional scheduling methods, whether analytical, heuristic, or metaheuristic (including GAs, Tabu search, simulated annealing, artificial neural networks, fuzzy logics), encounter great difficulties when they are applied to real-world situations. This is because they use simplified theoretical models and are essentially centralized in the sense that all computations are carried out in a central computing unit. The intelligent agent technologies, on the other hand, suggest an innovative, lightweight approach to scheduling problems. This essentially distributed approach is more flexible, efficient, and adaptable to real-world dynamic manufacturing environments.

\section{B. Agent-Based Approaches}

Within the past decade, a number of researchers have applied agent technology in attempts to resolve scheduling problems. Applications include manufacturing flow shop scheduling [18], [113] and job shop scheduling [49], [59], [60], transportation scheduling [27], power distribution scheduling [44], computing resource scheduling [31], meeting scheduling [100], medical test scheduling [38], and project management [54], [127]. An extensive bibliography on multiagent scheduling in manufacturing systems is compiled by Schiegg [88].

Agent-based approaches have several potential advantages for distributed manufacturing scheduling [95].

a) These approaches use parallel computation through a large number of processors, which may provide scheduling systems with high efficiency and robustness.

b) They can facilitate the integration of manufacturing process planning and scheduling.

c) They make it possible for individual resources to trade off local performance to improve global performance, leading to cooperative scheduling.

d) Resource agents may be connected directly to physical devices they represented for so as to realize real-time dynamic rescheduling (of course, not immediate rescheduling after any change in the working environment for the sake of system stability). It may therefore provide the manufacturing system with higher reliability and device fault tolerance.

e) Schedules are achieved by using mechanisms similar to those being used in manufacturing supply chains (i.e., negotiation rather than search). In this way, the manufacturing capabilities of manufacturers can be directly connected to each other and optimization is possible at the supply chain level, in addition to the shop floor level and the enterprise level.

f) Other techniques may be adopted at certain levels for decision-making, e.g., simulated annealing [48] and GAs [33], [96].

\section{Research Literature on Agent-Based Manufacturing Scheduling}

Research in agent-based manufacturing scheduling has been more active and has a richer literature base than that in agentbased manufacturing process planning. This section provides a detailed review in a structured way.

1) Earlier Attempts: Shaw may be the first person who proposed using agents in manufacturing scheduling and factory control. He suggested that a manufacturing cell could subcontract work to other cells through a bidding mechanism [89], [90]. Yet Another Manufacturing System (YAMS) [80] is another example of an early agent-based manufacturing system, wherein each factory and factory component is represented as an agent. Each individual agent has a collection of plans as well as knowledge about its own capabilities. The Contact Net protocol [104] is used for interagent negotiation.

2) Methodologies and Techniques: Different methodologies and techniques have been proposed, developed, and used in the literature for agent-based manufacturing scheduling.

a) CORTES [84], [111] uses micro-opportunistic techniques for solving the scheduling problem through a two-agent system, where each agent is responsible for scheduling a set of jobs and for monitoring a set of resources.

b) Baker [6] proposed a market-driven contract net for heterarchical agent-based scheduling. This agent architecture performs a type of forward/backward scheduling. 
c) Logistics Management System (LMS) [28] applies integration decision technologies to dispatch-scheduling in semiconductor manufacturing. It uses functional agents, one for each production constraint, and a judge agent to combine the votes of all the perspectives. Each agent partially models those aspects of the environment that are needed to satisfy its objective. Its uniqueness is a voting protocol for communication among agents.

d) Liu and Sycara [59] proposed a coordination mechanism called Constraint Partition and Coordinated Reaction (CP\&CR) for job shop constraint satisfaction. This system assigns each resource to a resource agent responsible for enforcing capacity constraints on the resource, and each job to a job agent responsible for enforcing temporal precedence and release-date constraints within each job. Moreover, a coordination mechanism called Anchor\&Ascend is proposed for distributed constraint optimization. Anchor\&Ascend employs an anchor agent to conduct local optimization of its subsolution and interacts with other agents that perform constraint satisfaction through CP\&CR to achieve global optimization [60].

e) In AARIA [79], the manufacturing capabilities (e.g., people, machines, and parts) are encapsulated as autonomous agents. Each agent seamlessly interoperates with other agents in and outside the factory boundary. AARIA used a mixture of heuristic scheduling techniques: forward/backward scheduling, simulation scheduling, and intelligent scheduling. Scheduling is performed by job, resource, and operation.

f) Miyashita [68] proposed an integrated architecture for distributed planning and scheduling using the repair-based methodology together with the constraint-based mechanism of dynamic coalition formation among agents. A prototype system called CAMPS is implemented, in which a set of intelligent agents try to coordinate their actions for satisfying planning/scheduling results by handling several intra- and interagent constraints.

g) Usher [116] presented an experimental approach for performance analysis of a multiagent system for job routing in job-shop settings: i) under various information levels for constructing and evaluating bids, and ii) under actual realtime process data for the negotiation process. Some simple but practical mechanisms are proposed and implemented.

h) Lu and Yih [61] proposed a framework that utilizes autonomous agents and weighted functions for distributed decision-making in elevator manufacturing and assembly. This system dynamically adjusts the priorities of subassemblies in the queue buffer of a cell by considering the real-time status of all subassemblies in the same order.

i) In [4], an agent-based scheduling system, incorporating game theoretic based agent cooperation, is presented to solve the $n$-job three-stage flexible flow shop scheduling problem. With scheduling task represented by a series of digraphs, MIP (mixed integer programming, minimizing makespan) is used by individual agents to schedule their jobs, and the final solution is reached by agent cooperation using game theory.
3) Approaches and Architectures: To satisfy the requirements for next-generation manufacturing systems, researchers have proposed and developed a number of approaches and architectures for agent-based manufacturing scheduling and control.

a) Burke and Prosser [10] described a distributed asynchronous scheduling (DAS) system. The DAS architecture consists of three types of entities: knowledge resources, agents, and a constraint maintenance system. The agents were originally developed as a multiagent heterarchy to represent only resources (O-agents). The final development includes agents for aggregations of resources (T-agents) and an agent for overseeing the whole scheduling process (S-agent). This final scheduling system organizes agents into a hierarchical architecture, in which the $\mathrm{S}$-agent assigns operations to the T-agents and the T-agents assign these operations further to O-agents, respectively. While DAS is able to make a correct schedule, however, it has no method for optimizing that schedule.

b) Scheduling in architecture for distributed dynamic manufacturing scheduling (ADDYMS) is decomposed into two levels [12]: the first level involves the assignment of a manufacturing work cell to a task, and the second consists of the determination of a local resource as well as other aspects, such as workers and tools, which may possibly be shared among a number of work cells. Corresponding to these two levels, there are two kinds of agents: site agents and resource agents. The system is composed of several connected site agents, each of which is in turn connected with its subsite agents and some local resource agents.

c) Lin and Solberg [58] showed how a market-like control model could be used for adaptive resource allocation and distributed scheduling. They modeled the manufacturing shop floor exactly like a market place, where each task agent enters the market carrying certain "currency" and bargains with each resource agent on which it can be proposed. At the same time, each resource agent competes with other agents to get a more "valuable" job. The market mechanism, using multiple-way and multiple-step negotiation, is incorporated to coordinate different agents, including part agents, resource agents, database agents, and communication agents.

d) Interrante and Rochowiak [43] proposed using active scheduling in the development of a multiagent architecture for dynamic manufacturing scheduling.

e) Murthy et al. [72] described an agent-based scheduling system based on the A-team architecture, in which functional agents generate, evaluate, improve, and prune a pool of candidate solutions. This system can be considered to be a blackboard system.

f) Kouiss et al. [49] proposed a multiagent architecture for dynamic job shop scheduling. Each agent represents a work center and performs a local dynamic scheduling by applying an adaptive dispatching rule. Depending on local and global considerations, a new selection of dispatching rule is carried out when a predefined event occurs. The selection method is improved through the optimization of the thresholds used to detect symptoms (events). Agents 
can also coordinate their actions to perform a global dynamic scheduling. However, a global agent is needed to detect the symptom of the shop floor.

g) Sousa and Ramos [106] proposed a dynamic scheduling system architecture composed of the holons representing tasks together with the holons representing manufacturing resources. The Contract Net protocol is adapted to handle temporal constraints and deal with conflicts. Sousa et al. [107] further proposed an extended Contract Net Protocol with constraints propagation for explicit representation of the precedence relationships between the operations of a task (with a cooperation phase between service providers). It shows some novelty compared with other variants of the Contract Net Protocol.

h) van Brussel et al. [117] proposed the PROSA reference architecture for holonic manufacturing systems (HMS). It uses a hierarchical system architecture to organize holons (similar to agents and implemented as agents in most HMS demonstrations as discussed below) and has been used as the basic architecture for implementing holonic manufacturing scheduling systems [19], [67], [63]. The architectural components include order holons, product holons, and resource holons.

i) Wang et al. [124] proposed a heterachical multiagent system and distributed ruler-based scheduling mechanism. The scheduling system is decomposed into three subsystems, i.e., management subsystem, resource subsystem, and part subsystem, and they are further decomposed into several units, which are assigned to several agents, i.e., management agents (MA), resource agents (RA), and part agents (PA). Rulers are encapsulated in agents and schedules are able to be established and executed in a parallel and distributed fashion.

j) An agent-based market-driven scheduling architecture was proposed by Yen and $\mathrm{Wu}$ [128] to integrate all kinds of existing manufacturing scheduling systems over the Internet. Each standalone scheduling system is endowed with agent feature by migrating the legacy system into an Internet scheduling agent. A network of heterogeneous scheduling agents collaborate or compete with each other for scheduling tasks using a market-driven protocolVickrey Auction.

k) Similarly, Goldberg et al. [34] uses a market-driven mechanism for task and resource allocation in a larger distributed, multirobot control and coordination architecture. Individual robot interacts with others to advance its selfinterest; however, the aggregate effect of a robot community can manifest mutual beneficiary effects.

4) Testbeds and Industrial Applications: Only a few testbeds and real industrial applications have been developed and reported in the literature. LMS [28] has been used in commercial production at IBM. The Manufacturing Scheduling and Control Testbed (MASCOT) [81] was a simulated testbed for manufacturing scheduling and control. It provides a communication infrastructure, a shared ontology, a shared interface based on that ontology, and a base set of realistic modules for the design, integration, and operation of agile enterprises. The TIE/Agent [40] was specially designed as an evaluation tool (testbed) for agentbased manufacturing systems (ABMS) based on its embedded ABMS model. It is a parallel simulator developed on Paragon supercomputer and the analytical results could clearly show the effects (marked by viability) of nonautonomous and autonomous factors on individual agents as well as the whole agent system. The shop floor agents' (SFA) project at NCMS [73] was focused on application of agent-based systems for shop floor scheduling and machine control. The project members include three manufacturers (AMP, GM, and Rockwell Automation/AllenBradley) and a software company (Gensym). The objective of the project is the design and development of agent-based systems to support three industrial scenarios, one sponsored by each of the manufacturing members of the project [82]. Cowling et al. [17] reported an application of intelligent agents to the dynamic scheduling in steel production.

Note that multiagent manufacturing systems are being considered to have substantial overlap with HMS [63]. The distributed information-processing mechanisms in individual holons and holonic systems are generally resulted in the implementation of multiagent systems [19] and the results of agent research community (including the standardization efforts like FIPA [26]) have been used to implement the concepts of HMS. This leads to the trend of merging the two academic research communitiesMAS and HMS [63]. More discussions on this topic can be found in [9] and [11]. Within the MAS/HMS community, a number of applications have been reported, including applications on agent-based manufacturing process planning and scheduling.

\section{APPROACHES to PROCESS PlanNING AND SCHEDULING INTEGRATION}

Traditionally, manufacturing process planning determines how a product should be manufactured by focusing on the pure geometric and technological requirements of tasks. It assigns machines, cutting tools, and cutting parameters to each process based on somewhat unrealistic assumptions, such as unlimited resources and an idle shop floor. Without the consideration of real-time machine workload and shop floor dynamics, the process plans made offline during the planning stage are often out of touch with shop floor operations at the time of task execution. Therefore, there is a need for the integration of manufacturing process-planning and scheduling systems for generating more realistic and effective plans to be used in the shop floor.

The topic of manufacturing process planning and scheduling integration has been the research focus of many researchers for more than ten years. The importance of the nonlinear process plan concept (NLPP, also called flexible process plan, alternative process plan, or multiple process plans) has been identified as a milestone for process planning and scheduling integration by almost all relevant publications.

Since no recent comprehensive survey is available in the area of manufacturing process planning and scheduling integration, this section has been expanded to include all the approaches in this area. It provides a comprehensive state-of-the-art review for both agent-based approaches as well as other traditional approaches. 
Note that some of the research projects or systems cited in this section may have been mentioned in Sections II-IV. However, they are classified into different categories and reviewed from different perspectives.

\section{A. Traditional Approaches}

1) Centralized Optimization Algorithms: Many researchers tried to combine process planning and scheduling as a single scheduling optimization problem. The complexity created by introducing alternative process plans and alternative resources to the already NP-hard scheduling problem has been analyzed in Section II. With the much more complex situations in realworld manufacturing process planning and dynamic shop floors, the combined solution space becomes so large that an effective optimization algorithm becomes an absolute necessity. As a result, a variety of optimization approaches have been studied and reported as follows.

a) The concurrent assignment algorithm proposed by Chen and Khoshnevis [16] uses a time-window scheme, an effective matrix, an operation matrix, and an optimal assignment algorithm to optimize the combination of multiple parts with multiple features and multiple machines.

b) Zijm [135] addressed the integration problem by searching alternatives for operations on the critical path based on graph theory, where the NLPPs are represented by graph theoretical expressions.

c) A branch-and-bound approach to optimize priorityweighted earliness of jobs scheduled in a mould manufacturing shop floor was proposed by Gan and Lee [30]. Some simple heuristics are used to speed up the algorithm. This algorithm together with the rescheduling mechanism was stated to be effective in terms of objective function and other performance measures.

d) Two algorithms were proposed and compared in [47]. The preprocessing algorithm combines the features of branch and bound and mixed integer programming techniques. Another algorithm is an iterative one based on a heuristic improvement method. The authors concluded that with the expansion of problem size, the preprocessing algorithm displays good quality results and consumes moderate computation time while the heuristic one is getting worse.

e) Lee and Kim [52] introduced a simulation-based GA approach, where a simulation model is used to compute the performance measures (predict scheduling) and a GA is used to evaluate and select the best process plan combination. The linear process plan obtained is then sent to a scheduling module to do the allocation task. In the scheduling approach proposed by Sugimura et al. [109], the sequence generation of machining features is realized by a GA and the selection of machining equipment is completed by dynamic programming for each machining sequence.

f) Palmer [77] applied simulated annealing (SA) to the integration problem and justified its optimization results. The cybernetic optimization by simulated annealing (COSA) [39] attempts to mitigate the slow convergence rate of gen- eral SA. The improved performance makes SA a candidate for solving the complex optimization of process planning and scheduling.

g) Tan [112] provided a literature review and many critiques toward the existing integration methods. He studied and extensively experimented various mathematical approaches to solve the problem, including linear mixed integer programming (LMIP), polynomial LMIP, Tabu search, and branch-and-bound procedure. Tan introduced a concept of cooperative solver that combines the strength of different algorithms to provide overall superior results with the capability of considering multiple optimization objectives.

Aiming at reducing the computation complexity of a big central optimization algorithm, some researchers tried to split the optimization problem into several steps, each with a distinguishing objective.

a) Brandimarte and Calderini [8] presented a hierarchical Tabu-search method on top of LMIP to deal with process selection and job shop scheduling. The problem is modeled with two objective functions, considering both the operation cost and the makespan.

b) Saygin and Kilic [87] proposed hierarchical integer linear programs with different objective functions to select alternative machines (shortest path) and alternative process plans (dissimilarity maximization), and to finish time scheduling of the obtained linear process plan. The three-step mathematical procedures make up an offline scheduling algorithm with good load balancing over potential resources. However, the rescheduling issue is only based on alternative machines. Some simple heuristics are used but cannot solve all the situations in dynamic shop floors.

2) Close Loop Optimization: Rather than listing alternative plans, some researchers argued that NLPP-oriented approaches for integrating process planning and scheduling are still in an offline mode. All the acceptable schedules made at the predictive scheduling stage are almost immediately subject to changes on the shop floor owing to the rescheduling factors, such as machine breakdowns, materials shortage, order cancellation, due date changes, and so on [87]. Although many of the systems adopting the above solutions were said to be responsive to shop floor disturbances, they cannot be called dynamic as process planning is performed before scheduling, with the same optimization algorithm invoked to do the initial scheduling and rescheduling. In the close loop process-planning (CLPP) approaches, process plans are created based on the dynamic feedback from the production scheduling or production control modules.

a) Mamalis et al. [62] used least cost and depth first search technique of the decision tree along with discrete event simulation to evaluate the suggested NLPPs. offline process plans and schedules are developed in the first phase of process planning and scheduling integration. In the second phase, the system works out eventual changes of the initially defined production conditions (mainly the utilization of factory resources), and regenerates alternative process 
plans. The authors called this phase an online integration of process planning and scheduling.

b) Within the ESPRIT project COMPLAIN, Kempenaers et al. [46] presented an architecture that integrates the ideas of manual/automated process planning and scheduling, together with the idea of feedback loops. The full closed loop solution includes production constraints as the feedback from the scheduling module to the process-planning module to facilitate the generation or regeneration of NLPPs. The COMPLAN project is the continuation of FLEXPLAN [51], which uses a Petri-net to model and analyze the flexibility provided by NLPPs (represented by AND/OR graphs).

3) Distributed Process-Planning (DPP) Approaches: The basic idea of DPP is to look upon the integration problem as a process-planning task. To integrate with scheduling, DPP advocates a more flexible plan scenario by separating the whole process to several steps.

a) Huang et al. [41] tried to separate the integration problem of process planning and scheduling into three phases: preplanning, pairing planning, and final planning. The preplanning is a technical manufacturing analysis of the product to identify machining requirements and constraints. Pairing planning matches the required job operations with the operation capabilities of the available production resources. The final planning prepares detailed manufacturing plans of the product for the selected equipment. The interaction of process planning and scheduling takes place in all three phases, starting from offline machine potential information support to machine group allocation and finally to specific machine selection within the machine group upon plan execution. From the viewpoint of process planning, the whole process plan is not deducted at once but progressively. This work is based on the results of the IPPM [130].

b) IDCPPS [14] is an integrated, distributed, and cooperative process-planning system. Similarly, the process-planning tasks are separated into three levels, namely, initial planning, decision-making, and detail planning. The responsibilities of each level are very similar to what has been presented by Huang et al. [41]. The results of these three steps are general process plans, a ranked list of near-optimal alternative plans and the final detailed linear process plans, respectively. The integration with scheduling is considered at each stage with process planning.

c) Wang and Shen [120] presented a new DPP methodology by integrating machining feature-based planning, function block-based control, and agent-based distributed decisionmaking. It proposes to use two-level decision-makingsupervisory planning and operation planning. The supervisory planning focuses on product data analysis, machine selection, and machining sequence planning, and the operation planning considers the detailed working steps of the machining operations inside each process plan and is accomplished by intelligent NC controllers. The function block-based integration architecture of DPP with manufacturing scheduling, execution, and control is discussed in more detail in [121].

\section{B. Agent-Based Approaches}

For complex optimization tasks, agent approaches provide a distributed intelligent solution by multiagent negotiation, coordination, and cooperation.

a) Gu et al. [35] proposed a multiagent system where the process routes and schedules of a part are accomplished through the contract net bids. Process-planning tasks, including STEP file parsing and interpretation, tolerance analysis, operation planning, setup planning, and machine, tool, and fixture selection, are all distributed to individual machine agents. There is no centralized process planner in this system although same planning techniques are applied to every machine. The task allocation and process alternative selection are achieved through the hierarchical bidding processes between machine agents and shop floor manager, between upper level machine agents and lower level machine agents, and between machine agents and tool agents, etc. A cost model is built to facilitate decision-making in the process of negotiation.

b) Denkena et al. [20] also presented a multiagent architecture to determine operation routes and schedules. Their approach separates the rough process-planning task as a centralized shop floor planner from the detailed process planning conducted through agent negotiations. The proposed multiagent planning architecture (centralized rough level process planning and decentralized planning and scheduling) builds a flexible, reliable, and fault-tolerant information logistics to enable supply chains, temporal logistic networks, or virtual enterprises.

c) IDCPPS [14] also uses agent-based techniques to implement its process planning and scheduling integration.

d) A cascading auction protocol [66] was proposed as a framework for integrating process planning and heterarchical shop floor control. The integration of the realtime online process planning (alleviating the selection of routing alternatives, resource alternatives, detail process planning, etc.), and shop floor control (resource allocation, scheduling, transportation, tooling, and fixturing) is accomplished progressively through a recursive auction process carried out in parallel among part management agent and multiple resource management agents. In this approach, the complete part production process plan could be constructed, including tooling, fixturing, and transportation plans.

Because of the autonomous and cooperative nature of agents, scheduling and process-planning functions can be integrated from either a higher level of separate functional decomposed agents [14] or a lower level of resource agents plus a coordinator [35].

\section{MAJOR DESIGN AND IMPLEMENTATION ISSUES}

\section{A. Agent Encapsulation}

Among different approaches used for agent encapsulation in agent-based manufacturing systems, two approaches are distinct: the functional decomposition approach and the physical decomposition approach. 
In the functional decomposition approach, software agents are used to encapsulate modules detailed to functions, such as order acquisition, process planning, scheduling, material handling, transportation management, and product distribution. There are no explicit relationships between software agents and physical entities. In the physical decomposition approach, software agents are used to represent entities in the physical world, such as workers, machines, tools, fixtures, products, parts, features, operations, etc. There exist explicit relationships between software agents and physical entities.

The functional decomposition approach tends to share many state variables across different functions. Separate agents must share many state variables, thus leading to problems of consistency and unintended interactions. The physical decomposition approach naturally defines distinct sets of state variables that can be managed efficiently by individual agents with limited interactions, but it needs a large number of resource-related agents, thus leading to other problems, such as communication overhead and complex agent management. However, the functional decomposition approach is very useful to integrate existing systems (e.g., CAD tools, MRP systems, databases, etc.) so as to resolve legacy problems.

Corresponding to the two distinct approaches for agent encapsulation, two types of agent-based distributed manufacturing scheduling systems can be distinguished according to the following characteristics.

a) Scheduling is an incremental search process that can involve backtracking. Agents, responsible for scheduling orders, perform local incremental searches for their orders and may consider multiple resources. The global schedule is obtained through the merging of local schedules. This is very similar to centralized scheduling.

b) An agent represents a single resource (e.g., a work cell, a machine, a tool, a fixture, a worker, etc.) and is responsible for scheduling this resource. This agent may negotiate with other agents to carry out the overall scheduling.

Examples of the second type of scheduling systems can be found in [10], [28], [66], [72], [84], [101], [106], and [124]. Most agent-based manufacturing scheduling systems proposed and developed in literature use the second approach.

Things become complex when considering process-planning tasks or their integrated systems. Most agent-based processplanning systems (including scheduling integrated systems) follow the functional decomposition of agents [22], [55], [99], [132], [134]. How to build multiagent systems to solve complex applications using the physical agent decomposition approach, or a combination of both approaches, is a possible future research direction.

\section{B. Agent Modeling}

In agent-based manufacturing process-planning and scheduling systems, bidding-based negotiations or market-like approaches are commonly used. This section will address the decision schemes that an individual agent should have to realize effective agent-based scheduling. Discussions in the following paragraphs are also related to the systems using the physical de- composition approach, rather than the functional decomposition approach.

This section does not discuss in detail the different kinds of agent architectures. A detailed discussion on desired characteristics and commonly used modules of agents in agent-based collaborative design and manufacturing systems can be found in [94].

In agent-based manufacturing scheduling systems, the applied agent negotiation protocols require individual agents to reply to the incoming offers, to compete, and to negotiate or to bargain with other agents. As a result, rich knowledge bases and powerful learning and reasoning mechanisms are very important. Each agent should have at least knowledge about the capability, availability, and cost of the physical resource (e.g., a machine) represented by itself. Some sophisticated agents need to have knowledge about other agents in the system, the products to be manufactured, and know-how (historical experience, successful cases), etc.

The decision scheme of an individual agent depends primarily on two aspects: coordination or negotiation mechanisms used by the multiagent system and its local decision-making mechanisms based on knowledge. For example, a Contract Net protocol needs each individual agent to reply to an offer with requested information such as cost, start time, processing time, etc. [80]. A game-theory-based multiagent system needs agents to follow game rules [4], [36]. While a multiagent system implemented with a conversation scheme will need each agent to follow the conversation policies [57]. Local decision-making may use rule-based and case-based mechanisms reasoning on top of the knowledge the agent possesses. To update an agent's knowledge, learning mechanisms are needed. Such learning mechanisms may range from case-based reasoning to neural network and fuzzy logic-based reasoning.

\section{System Structures}

Agent system architectures provide the organizing frameworks within which agents are designed and constructed. The various system architectures proposed in the literature for agent-based manufacturing process planning and scheduling can be classified into three categories: hierarchical, federate, and autonomous.

A typical modern manufacturing enterprise consists of a number of physically distributed, semiautonomous units, each with a degree of control over local resources and with different information requirements. In this situation, many practical agent-based industrial applications still use the hierarchical architecture, even though this has the disadvantages introduced by centralized systems. In fact, agent-based distributed manufacturing process-planning and scheduling systems using functional decomposition (each agent represents a function or a department in a traditional system) usually have this kind of hierarchical structure. Examples can be found in HMS [106], ADDYMS [12], DAS [10], and LMS [28].

Because of the serious problems caused by the central characteristics associated with hierarchical structure, the federated architectures are increasingly being considered as a 
compromise solution for industrial agent-based applications, especially for large-scale engineering applications. Federated multiagent architectures are able to coordinate multiagent activities via facilitation or mediation as a means of reducing communication overhead and ensuring system stability and scalability. A fully federated agent-based system has no explicit shared facility for storing active data; rather, the system stores all data in local databases and handles updates and changes through message passing. The federated approach promises to be a good foundation upon which to develop open, scalable multiagent system architectures.

Different federated architectures have been proposed in the literature for agent-based manufacturing systems. Four approaches are dominant: facilitators, brokers, matchmakers, and mediators.

In the facilitator approach, several related agents are combined into a group. Communication between agents takes place always through an interface called facilitator. Each facilitator is responsible for providing a gateway between a local collection of agents and remote agents, usually by providing two main services: 1) routing outgoing messages to the appropriate destinations; 2) translating incoming messages for consumption by its local agents. This approach has been widely used in agentbased collaborative design systems [94].

Brokers (also called broker agents) are similar to facilitators in some aspects, such as monitoring and notification. The difference between a facilitator and a broker is that a facilitator is responsible only for a designated group of agents, whereas a broker can be contacted by any agent dynamically in case they need services to finish their tasks. The matchmaking mechanism is a superset of the brokering mechanism, since it uses the brokering mechanism to match agents. Yellow page agents and Directory Facilitators proposed by Foundation of Intelligent Physical Agents (FIPA) [26] are similar to matchmakers. Directory Facilitator has been used in the iShopFloor project [93] for providing yellow page-like services to all other agents.

Mediator approach is another type of federated architecture. In addition to the functions of a facilitator and a broker, a mediator assumes the role of system coordinator by promoting cooperation and learning among intelligent agents. Applications using mediators in intelligent manufacturing systems can be found in [64], [65], [76], [91], and [93].

In the third structure in the category (autonomous multiagent approach), each autonomous agent usually has the following characteristics: 1) it is not controlled or managed by any other software agents or human beings; 2) it can communicate/interact directly with any other agents in the system and also with other external systems; 3) it has knowledge about other agents and its environment; and 4) it has its own goals and an associated set of motivations. Examples can be found in [23], [58], [79], [83], and [128]. However, the autonomous approach is only well suited for developing distributed systems consisting of a small number of agents.

In the mediator architecture, a static or dynamic hierarchy is imposed for every specific task, which can provide computational simplicity and manageability. This type of architecture is quite suitable for developing distributed manufacturing process- planning and scheduling systems, which are complex, dynamic, and composed of a large number of resource agents. A combination of above-mentioned three approaches called "hybrid approach" was proposed in MetaMorph II [91] for developing more flexible, modular, scalable, and dynamic manufacturing systems.

\section{Coordination and Negotiation}

When a system uses the functional decomposition approach, it is similar to traditional integrated systems. Such a system usually needs a predefined coordination mechanism. Discussions in the following paragraphs are therefore more related to the systems using the physical decomposition approach.

Negotiation protocols are used in most agent-based manufacturing scheduling systems for resource allocation. The Contract Net Protocol or its modified versions are the most commonly utilized, although some other protocols such as the voting mechanism by Fordyce and Sullivan [28] have also been considered. The Contract Net Protocol was first proposed by Smith [104] and demonstrated on a distributed sensing system. To summarize briefly, an agent (manager) having some work to subcontract broadcasts its offer and waits for other agents (contractors) to send back their bids. After some delay, the best offers are retained and contracts are allocated to one or more contractors that process the subtask. The Contract Net Protocol is a coordination method for task allocation, providing dynamic allocation and natural load balancing. However, as the number of nodes increases, the communication workload on the network soars, which may lead to a situation where agents spend more time in processing messages than doing actual work. Thus, various improvements to the basic contract net approach have been proposed, such as the following:

1) sending offers to a limited number of nodes, instead of broadcasting them;

2) anticipating offers, i.e., contractors send bids in advance;

3) varying the time when commitment is decided;

4) allowing de-commitment (breaking commitments);

5) allowing several agents to answer as a group (coalition formation);

6) introducing priorities for solving tasks.

The CNP-based bidding mechanism used in manufacturing scheduling can be part-oriented [23], [58], resource-oriented [6], [12], or bidirectional [83].

In basic CNP approaches, the choice of a contractor is done by comparing bids corresponding to a particular offer using whatever mechanisms that are relevant to the problem. In some more complex cases, e.g., involving de-commitment [85], penalties are introduced thus bringing the approach closer to a market-like approach [71]. Shen et al. [91] presented a promising approach by combining Contract-Net-based negotiation and mediatorbased coordination for agent-based manufacturing scheduling and rescheduling.

Although Contact Net and its variants are usually used as negotiation protocols in most agent-based scheduling systems, market-based approaches are becoming more and more popular. Market-based or like protocols use the so-called bargaining 
process or auction process, which is also simple and easy to use. Market-based or like approaches have recently been used in a number of agent-based scheduling systems [6], [34], [54], [58], [66].

Some recent research work is also showing some advantages by combining agent-based approaches with other techniques such as GAs, neural networks, fuzzy logic [86], and some mathematical modeling methods. Market-like or biddingbased methods emphasize system flexibility and responsiveness over optimality of solutions, and are, therefore, more suitable for dynamic rescheduling. Search methods, like GAs and simulated annealing, focus more on the optimality of solutions and are, therefore, more suitable for advance scheduling. A combination of these methods can be used in intelligent shop floors requiring both advance scheduling and dynamic scheduling. For example, Daouas et al. [18] proposed combining agent-based negotiation with simulated annealing search, while Shen [93] proposes to combine agent-based negotiation with GA-based search for scheduling optimization.

Sycara et al. [106] proposed a different approach using "texture measures," where all agents share a common information base, called coordination agent. This approach requires each agent to compute its own texture measure and submit it to the coordination agent, and then read the integrated texture measure to make its own decision. After individual agents make their decisions, they submit their solutions to the coordination agent that in turn regulates the possible conflicts. This approach could be used for agents to predict possible conflicts, but not to eliminate conflicts.

Some researchers have also realized the game-like nature of independent scheduling decisions, and try to use game theory to make their agents smarter [36].

Very recently, a new adaptive negotiation approach has been proposed by our group to address complex negotiation situations [122].

\section{RESEARCH OPPORTUNITIES AND CHALLENGES}

This paper provides a state-of-the-art survey of research on agent-based manufacturing process planning and scheduling. Based on this survey, as well as on our first-hand research and development experience in this area, future research opportunities, as well as challenges, are identified as follows.

1) Theoretical investigation of methodologies needed for agent-based distributed manufacturing process planning and scheduling. Theoretical investigation of methodologies, including implementation methodology, is needed to consolidate the current research results in this area and facilitate the implementation of real industrial applications.

2) Negotiation mechanisms, protocols, frameworks. As most agent-based manufacturing process planning and scheduling use bidding-based or market-like negotiation mechanisms/protocols, research and development of more powerful negotiation mechanisms and protocols are needed. Combinatorial market-based negotiation protocols are of much interest in the near future. Advanced negotiation frameworks are to be investigated, e.g., adaptive nego- tiation frameworks together with combined negotiation techniques [97].

3) Integration of process planning, manufacturing scheduling, and control. Agent-based approaches provide a natural way to integrate manufacturing process planning, scheduling, and execution control. It also provides the possibility of simultaneous optimization of process planning and manufacturing scheduling. However, it increases significantly the complexity of the problem. Much more effort needs to be devoted to this research topic, including complexity analysis and formal modeling of such integration.

4) Integration with real-time information. Without considering real-time information including changing customer requirements and shop floor dynamics, the process plans and manufacturing schedules generated offline may become invalid at the time of task execution. Real-time dynamic scheduling has been an important research topic [49], [106], but significant efforts are needed before solutions can be widely used in industry.

5) Integration of agent-based approaches with other approaches. As mentioned early in the paper, market-like or bidding-based methods emphasize system flexibility and responsiveness over optimality of solutions. They are more suitable for dynamic rescheduling. While search methods like GAs and simulated annealing focus more on the optimality of solutions and are, therefore, more suitable for advance scheduling. It is interesting to integrate these two kinds of methods into powerful manufacturing process-planning and scheduling systems, e.g., integration of agent-based negotiation with GA-based search [96], with artificial neural-network-based learning or fuzzy logic-based learning, with simulated annealingbased optimization [18], [79], with Petri-net/Color Petrinet-based coordination [57], [123], with heuristic search methods [79], and with matrix-based methods [1].

6) Combination of individual problem-solving and coordination/negotiation schemes. Obviously, there is a tradeoff between individual problem solving at the individual agent level and the coordination/negotiation scheme at the system level. A big challenge is how to combine them using the integration approaches mentioned above.

7) Integration with function blocks approach. The function blocks specification [42], a powerful modeling approach in the industrial process control field, has been considered as a critical media to integrate data, events, and decisionmaking processes of various manufacturing activities. Integration of agent-based approaches with function blocks seems to be a promising solution for the integration of manufacturing process planning, scheduling, and execution control [121].

8) Benchmark for agent-based distributed manufacturing process-planning and scheduling problems. Benchmarks are needed to evaluate various systems proposed and developed using different approaches [13], as well as to compare with systems implemented using traditional techniques/approaches. 
9) Assurance of system security. System security is not the focus of this paper, but a major concern of implementing Internet-enabled manufacturing systems, which is the assurance that proprietary information owned by the organization or information about company operations is available only to authorized individuals or organizations. This problem must be solved before agent-based manufacturing process-planning and scheduling systems can be implemented in industrial settings [126].

\section{CONCLUDING REMARKS}

It is becoming clear that agent-based approaches offer many advantages for distributed manufacturing process-planning and scheduling systems: modularity, reconfigurability, scalability, upgradeability, and robustness (including fault recovery). The results achieved so far in the agent research community provide excellent motivation for further development of solutions in this area. Moreover, at present, there are no other ways to solve these complex problems. However, whether the potential advantages of agent-based approaches can actually be realized in industrial systems will depend on the selection of a suitable system architecture for agent organization and an appropriate approach for agent encapsulation; on the design and implementation of effective mechanisms and protocols for communication, cooperation, coordination, and negotiation; and on the design and implementation of advanced internal architectures and efficient decision schemes of individual agents.

\section{REFERENCES}

[1] M. Akbulut and S. V. Kamarthi, "Agent-based scheduling system to achieve agility," presented at the SPIE Int. Conf. Network Intelligence: Internet Based Manufacturing, Boston, MA, 2000.

[2] L. Alting and H. C. Zhang, "Computer aided process planning: The state-of-the-art survey," Int. J. Prod. Res., vol. 27, no. 4, pp. 553-605, 1989.

[3] B. Arezoo, K. Ridgway, and A. M. A. Al-Ahmari, "Selection of cutting tools and conditions of machining operations using an expert system," Comput. Ind., vol. 42, pp. 43-60, 2000.

[4] A. Babayan and D. He, "A distributed scheduling methodology for a two-machine flowshop using cooperative interaction via multiple coupling agents," Int. J. Prod. Res., vol. 42, no. 4, pp. 777-7104, 2004.

[5] T. P. Bagchi, Multiobjective Scheduling by Genetic Algorithms. Norwell, MA: Kluwer, 1999.

[6] A. D. Baker "Manufacturing control with a market-driven contract net," Ph.D. thesis, Rensselaer Polytechnic Inst., Troy, NY, 1991.

[7] R. M. Boogert, H. J. Kals, and F. J. Van Houten, "Tool paths and cutting technology in computer-aided process planning," Int. J. Adv. Manuf. Technol., vol. 11, pp. 186-197, 1996.

[8] P. Brandimarte and M. Calderini, "A hierarchical bicriterion approach to integrated process plan selection and job shop scheduling," Int. J. Prod. Res., vol. 33, no. 1, pp. 161-181, 1995.

[9] R. W. Brennan and D. H. Norrie, "Agents, holons and function blocks: Distributed intelligent control in manufacturing," J. Appl. Syst. Stud., vol. 2, no. 1, pp. 1-19, 2001.

[10] P. Burke and P. Prosser, "The distributed asynchronous scheduler," in Intelligent Scheduling, M. Zweben and M. S. Fox, Eds. San Francisco, CA: Morgan Kaufmann, 1994, pp. 309-339.

[11] S. Bussmann, "An agent-oriented architecture for holonic manufacturing control," in Proc. 1st Int. Workshop Intell. Manuf. Syst., Lausanne, Switzerland, 1998, pp. 1-12.

[12] J. Butler and H. Ohtsubo, "ADDYMS: Architecture for distributed dynamic manufacturing scheduling," in Artificial Intelligence Applications in Manufacturing, A. Famili, D. S. Nau, and S. H. Kim, Eds. Menlo Park, CA: AAAI Press, 1992, pp. 199-214.

[13] S. Cavalieri, L. Bongaerts, M. Macchi, M. Taisch, and J. Wyns, "A benchmark framework for manufacturing control," in Proc. 2nd Int. Workshop Intell. Manuf. Syst., Leuven, Belgium, 1999, pp. 225-236.

[14] F. T. S. Chan, J. Zhang, and P. Li, "Modelling of integrated, distributed and cooperative process planning system using an agent-based approach," in Proc. Inst. Mech. Eng., Part B-J. Eng. Manuf., vol. 215, no. B10, 2001, pp. 1437-1451.

[15] P. T. Chang and C. H. Chang, "An integrated artificial intelligent computeraided process planning system," Int. J. Comput. Integr. Manuf., vol. 13, no. 6, pp. 483-497, 2000.

[16] Q. Chen and B. Khoshnevis, "Scheduling with flexible process plans," Prod. Planning Control, vol. 4, no. 4, pp. 333-343, 1993.

[17] P. I. Cowling, D. Ouelhadj, and S. Petrovic, "A multi-agent architecture for dynamic scheduling of steel hot rolling," J. Intell. Manuf., vol. 14, no. 5, pp. 457-470, 2003.

[18] T. Daouas, K. Ghedira, and J. P. Muller, "How to schedule a flow shop plant by agents," in Applications of Artificial Intelligence in Engineering. Billerica, MA: Computational Mechanics Inc., 1995, pp. 73-80.

[19] S. M. Deen, Agent-Based Manufacturing - Advances in the Holonic Approach. Berlin, Germany: Springer-Verlag, 2003.

[20] B. Denkena, H. K. Tonshoff, M. Zwick, and P. O. Woelk, "Process planning and scheduling with multiagent systems," in Knowledge and Technology Integration in Production and Services-Balancing Knowledge and Technology in Product and Service Life Cycle. Norwell, MA: Kluwer, 2002, pp. 339-348.

[21] C. R. Devireddy and K. Ghosh, "Feature-based modeling and neural network-based CAPP for integrated manufacturing," Int. J. Comput. Integr. Manuf., vol. 12, no. 1, pp. 61-74, 1999.

[22] D. Dornfeld, P. K. Wright, F. C. Wang, P. Sheng, J. Stori, V. Sundararajan, N. Krishnan, and C. H. Chu, "Multi-agent process planning for a networked machining service," in Tech. Paper NAMRC XXVII, vol. MS99175, 1999, pp. 1-6.

[23] N. A. Duffie and R. S. Piper, "Non-hierarchical control of manufacturing systems," J. Manuf. Syst., vol. 5, no. 2, pp. 137-139, 1986.

[24] K. O. Edalew, H. S. Abdalla, and R. J. Nash, "A computer-based intelligent system for automatic tool selection," Mater. Design, vol. 22, pp. 337-351, 2001.

[25] H. A. Elmaraghy, "Evolution and future perspectives on CAPP," CIRP Ann. (Keynote), vol. 42, no. 2, pp. 739-751, 1993.

[26] FIPA (Foundation of Intelligent Physical Agents). [Online]. Available: http://www.fipa.org/

[27] K. Fischer, J. P. Muller, M. Pischel, and D. Schier, "A model for cooperative transportation scheduling," in Proc. ICMAS' 95, San Francisco, CA, 1995, pp. 109-116.

[28] K. Fordyce and G. G. Sullivan, "Logistics management system (LMS): Integrating decision technologies for dispatch scheduling in semiconductor manufacturing," in Intelligent Scheduling, M. Zweben and M. S. Fox, Eds. San Francisco, CA: Morgan Kaufmann, 1994, pp. 473-516.

[29] S. French, Sequencing and Scheduling: An Introduction to the Mathematics of the Job-Shop. New York: Wiley, 1982.

[30] P. Y. Gan and K. S. Lee, "Scheduling of flexible-sequenced process plans in a mould manufacturing shop," Int. J. Adv. Manuf. Technol., vol. 20, pp. 214-222, 2002.

[31] A. Glockner and J. Pasquale, "Coadaptive behavior in a simple distributed job scheduling," IEEE Trans. Syst., Man, Cybern., vol. 23, no. 3, pp. $902-$ 907, May-Jun. 1993.

[32] F. W. Glover and M. Laguna, Tabu Search. Norwell, MA: Kluwer, 1997.

[33] D. Goldberg, Genetic Algorithms in Search, Optimization and Machine Learning. Reading, MA: Addison-Wesley, 1989.

[34] D. Goldberg, V. Cicirello, and M. B. Dias, "Task allocation using a market-based planning mechanism," in Proc. Int. Conf. AAMAS'03, vol. 2, Melbourne, Australia, 2003, pp. 996-997.

[35] P. Gu, S. Balasubramanian, and D. H. Norrie, "Bidding-based process planning and scheduling in a multi-agent system," Comput. Ind. Eng., vol. 32, no. 2, pp. 477-496, 1997.

[36] Z. Guan, M. Lei, B. Wu, Y. Wu, and S. Yang, "Application of decentralized cooperative problem solving in dynamic flexible scheduling," in Proc. SPIE, Bellingham, WA, 1995, pp. 179-183.

[37] K. Hashmi, M. A. El Baradie, and M. Ryan, "Fuzzy logic based intelligent selection of machining parameters," Comput. Ind. Eng., vol. 35, no. 3-4, pp. 571-574, 1998.

[38] L. T. Herren, B. L. Robey, and P. K. Fink, "Multi-agent knowledge-based system for planning and scheduling medical tests," in Proc. IEEE Symp. Comput-Based Med. Syst., Jun. 13-16, 1993, pp. 159-164. 
[39] J. W. Herrmann, M. Eleischer, E. Lin, V. Mathur, and J. Glasser, "Affordable space systems manufacturing: Intelligent synthesis technology, process planning, and production scheduling," presented at the ASME DETC/CIE 2001, Pittsburgh, US, 2001, DETC2001/ CIE-21281.

[40] C. Y. Huang and S. Y. Nof, "Evaluation of agent-based manufacturing systems based on a parallel simulator," Comput. Ind. Eng., vol. 43, pp. 529$552,2002$.

[41] S. H. Huang, H. C. Zhang, and M. L. Smith, "A progressive approach for the integration of process planning and scheduling," IEE Trans., vol. 27, no. 4, pp. 456-464, 1995.

[42] International Electrotechnical Commission (IEC), IEC 61499: function blocks for industrial-process measurement and control systems (part-1: architecture), IEC TC65/WG6 Draft, 2000.

[43] L. D. Interrante and D. M. Rochowiak, "Active rescheduling and collaboration in dynamic manufacturing systems," Concurrent Eng.: Res. Appl., vol. 2, no. 2, pp. 97-105, 1994.

[44] N. R. Jennings, J. M. Corera, and I. Laresgoiti, "Developing industrial multi-agent systems," in Proc. ICMAS' 95, 1995, pp. 423-430.

[45] J. Y. Jung and R. S. Ahluwalia, "Prismatic part feature extraction and feature-based tool path selection," J. Des. Manuf., vol. 3, no. 1, pp. 1-19, 1993.

[46] J. Kempenaers, J. Pinte, J. Detand, and J. P. Kruth, “A collaborative process planning and scheduling system," Adv. Eng. Softw., vol. 25, no. 1, pp. 3-8, 1996.

[47] K. H. Kim and P. J. Egbelu, "Scheduling in a production environment with multiple process plans per job," Int. J. Prod. Res., vol. 37, no. 12, pp. 2725-2753, 1999.

[48] S. Kirkpatrick, C. D. Gelatt, and M. P. Vecchi, "Optimization by simulated annealing," Science, vol. 220, pp. 671-680, 1983.

[49] K. Kouiss, H. Pierreval, and N. Mebarki, "Using multi-agent architecture in FMS for dynamic scheduling," J. Intell. Manuf., vol. 8, no. 1, pp. 41-47, 1997.

[50] S. Kornienko, O. Kornienko, and P. Levi, "Flexible manufacturing process planning based on the multi-agent technology," in Proc. 21 st IASTED Int. Conf. Applied Informatics, Innsbruck, Austria, Feb. 10-13, 2003, pp. 156161.

[51] J. P. Kruth and J. Detand, "A CAPP system for nonlinear process plans," Ann. CIRP, vol. 41, no. 1, pp. 489-492, 1992.

[52] H. Lee and S. S. Kim, "Integration of process planning and scheduling using simulation based genetic algorithms," Int. J. Adv. Manuf. Technol., vol. 18, no. 8, pp. 606-590, 2001

[53] K. Y. Lee and M. Y. Jung, "Flexible process sequencing using Petri net theory," Comput. Ind. Eng., vol. 28, no. 2, pp. 279-290, 1995.

[54] Y. Lee, S. R. Kumara, and K. Chatterjee, "Multiagent based dynamic resource scheduling for distributed multiple projects using a market mechanism," J. Intell. Manuf., vol. 14, no. 5, pp. 471-484, 2003.

[55] M. Lim and Z. Zhang, "APPSS-An agent-based dynamic process planning and scheduling system," in Proc. Volume from the IFAC Workshop, 2000 , pp. 51-56.

[56] T. Lim, J. Corney, J. M. Ritchie, and D. E. R. Clark, "Optimizing tool selection," Int. J. Prod. Res., vol. 39, no. 6, pp. 1239-1256, 2001.

[57] F. Lin, D. H. Norrie, W. Shen, and R. Kremer, "Conversation specification-A schema-based approach to specifying conversation policies," in Issues in Agent Communication, F. Dignum and M. Greaves, Eds. Lecture Notes in Computer Science, vol. 1916, New York: SpringerVerlag, 2000, pp. 193-204.

[58] G. Y. J. Lin and J. J. Solberg, "Integrated shop floor control using autonomous agents," IIE Trans.: Des. Manuf., vol. 24, no. 3, pp. 57-71, 1992.

[59] J. Liu and K. P. Sycara, "Distributed problem solving through coordination in a society of agents," presented at the 13th Int. Workshop on DAI, 1994.

[60] — " "Exploiting problem structure for distributed constraint optimization," in Proc. 1st Int. Conf. Multi-Agent Systems, 1995, pp. 246-253.

[61] T. P. Lu and Y. Yih, "An agent-based production control framework for multiple-line collaborative manufacturing," Int. J. Prod. Res., vol. 39, no. 10 , pp. $2155-2176,2001$

[62] A. G. Mamalis, I. Malagardis, and K. Kanbouris, "online integration of process planning module with production scheduling," Int. J. Adv. Manuf. Technol., vol. 12, no. 5, pp. 330-338, 1996.

[63] V. Mařik, D. McFarlane, and P. Valckenaers, Holonic and Multi-Agent Systems for Manufacturing (1st Int. Conf. on Industrial Applications of Holonic and Multi-Agent Systems). Berlin, Germany: Springer-Verlag, 2003.
[64] F. Maturana, W. Shen, and D. H. Norrie, "MetaMorph: An adaptive agentbased architecture for intelligent manufacturing,"Int.J.Prod. Res., vol. 37 , no. 10, pp. 2159-2174, 1999.

[65] F. Maturana, S. Balasubramanian, and D. H. Norrie, "A multi-agent approach to integrated planning and scheduling for concurrent engineering," in Proc. Int. Conf. Concurrent Engineering: Research and Applications, Toronto, ON, Canada, 1996, pp. 272-279.

[66] P. McDonnell, G. Smith, S. Joshi, and S. R. T. Kumara, "A cascading auction protocol as a framework for integrating process planning and heterarchical shop floor control," Int. J. Flexible Manuf. Syst., vol. 11, no. 1, pp. 37-62, 1999.

[67] D. C. McFarlane and S. Bussmann, "Developments in holonic production planning and control," Prod. Planning Control, vol. 11, no. 6, pp. 522-536, 2000.

[68] K. Miyashita, "CAMPS: A constraint-based architecture for multi-agen planning and scheduling," J. Intell. Manuf., vol. 9, no. 2, pp. 147-154 1998.

[69] L. Monostori, Z. J. Viharos, and S. Markos, "Satisfying various requirements in different levels and stages of machining using one general ANNbased process model," J. Mater. Process. Technol., vol. 107, pp. 228-235, 2000 .

[70] N. Morad and A. Zalzala, "Genetic algorithms in integrated process planning and scheduling," J. Intell. Manuf., vol. 6, pp. 169-179, 1999.

[71] T. Mullen and M. P. Wellman, "Some issues in the design of marketoriented agents," in Intelligent Agents II: Agent Theories, Architectures, and Languages, M. Wooldridge, J. P. Müller, and M. Tambe, Eds. Lecture Notes in Artificial Intelligence, vol. 1037, New York: Springer-Verlag, 1996, pp. 283-298.

[72] S. Murthy, R. Akkiraju, J. Rachlin, and F. Wu, "Agent-based cooperative scheduling," in Proc. AAAI Workshop Constrains and Agents, 1997 pp. $112-117$.

[73] NCMS, "Shop floor agents," Nat. Center for Manuf. Sci. Ann Arbor, MI, Tech. Rep., 1998.

[74] B. W. Nieble, "Mechanized process selection for planning new designs," presented at the ASTME, 1965, Paper 737.

[75] S. K. Ong and A. Y. C. Nee, "Fuzzy-set-based approach for concurrent constraint setup planning," J. Intell. Manuf., vol. 7, no. 2, pp. 107-120, 1996.

[76] D. Ouelhadj, C. Hanachi, and B. Bouzouia, "Multi-agent system for dynamic scheduling and control in manufacturing cells," in Working Notes of the Agent-Based Manufacturing Workshop, Minneapolis, MN, 1998 pp. 96-105.

[77] G. J. Palmer, "A simulated annealing approach to integrated production scheduling," J. Intell. Manuf., vol. 7, no. 3, pp. 163-176, 1996.

[78] H. G. Park and J. M. Baik, "Enhancing manufacturing product development through learning agent system over internet," Comput. Ind. Eng., vol. 37, pp. 117-120, 1999.

[79] V. D. Parunak, A. D. Baker, and S. J. Clark, "The AARIA agent architecture: An example of requirements-driven agent-based system design," presented at the 1st Int. Conf. Autonomous Agents, Marina del Rey, CA, 1997.

[80] V. D. Parunak, "Manufacturing experience with the contract net," in Distributed Artificial Intelligence, M. N. Huhns, Ed. New York: Pitman, 1987, pp. 285-310.

[81] _ "MASCOT: A virtual factory for research and development in manufacturing scheduling and control," Ind. Technol. Inst., Tech. Memo. 93-02, 1993.

[82] — "Workshop report: Implementing manufacturing agents," Nat. Center for Manuf. Sci., Ann Arbor, MI, Tech. Rep., 1996.

[83] A. Saad, G. Biswas, K. Kawamura, M. E. Johnson, and A. Salama, "Evaluation of contract net-based heterarchical scheduling for flexible manufacturing systems," in Proc. 1995 IJCAI' 95, Workshop Intell. Manuf., Montreal, QC, Canada, 1995, pp. 310-321.

[84] N. Sadeh and M. S. Fox, "CORTES: An exploration into micro-opportunistic job-shop scheduling," presented at the Workshop on Manufacturing Production Scheduling (IJCAI-89), Detroit, 1989.

[85] T. Sandholm and V. Lesser, "Issues in automated negotiation and electronic commerce: Extending the contract net framework," in Proc. 1st Int. Conf. Multi-Agent Systems, 1995, pp. 328-335.

[86] J. Sauser, G. Suelmann, and H. J. Appelrath, "Multi-site scheduling with fuzzy concepts," Int. J. Approx. Reason., vol. 19, pp. 145-160, 1998. 
[87] C. Saygin and S. E. Kilic, "Integrating flexible process plans with scheduling in flexible manufacturing systems," Int. J. Adv. Manuf. Technol., vol. 15 , no. 4, pp. 268-280, 1999.

[88] P. Schiegg, Bibliography on Multi-Agent Scheduling in Manufacturing Systems. [Online]. Available: http://farm.ecs.umass.edu/pschiegg/bib/ lit.html

[89] M. J. Shaw, "Dynamic scheduling in cellular manufacturing systems: A framework for networked decision making," J. Manuf. Syst., vol. 7, no. 2, pp. 83-94, 1988.

[90] M. J. Shaw and A. B. Whinston, "Distributed planning in cellular flexible manufacturing systems," Manage. Inform. Res. Center, Purdue Univ., West Lafayette, IN, Tech. Rep., 1983.

[91] W. Shen, D. Xue, and D. H. Norrie, "An agent-based manufacturing enterprise infrastructure for distributed integrated intelligent manufacturing systems," in Proc. 3rd Int. Conf. Practical Application of Intelligent Agents and Multi-Agents (PAAM98), London, U.K., 1998, pp. 533-548.

[92] W. Shen and D. H. Norrie, "Agent-based systems for intelligent manufacturing: A state-of-the-art survey," Knowl. Inf. Syst., vol. 1, no. 2, pp. 129-156, 1999.

[93] W. Shen, S. Lang, L. Korba, L. Wang, and B. Wong, "Reference architecture for internet-based intelligent shop floors," in Proc. SPIE Int. Conf. Network Intelligence: Internet Based Manufacturing, vol. 4208, Boston, MA, 2000, pp. 63-71.

[94] W. Shen, D. H. Norrie, and J. P. Barthès, Multi-Agent Systems for Concurrent Intelligent Design and Manufacturing. London, U.K.: Taylor \& Francis, 2001.

[95] W. Shen, "Distributed manufacturing scheduling using intelligent agents," IEEE Expert/Intell. Syst., vol. 17, no. 1, pp. 88-94, Jan./Feb. 2002.

[96] _ " "Genetic algorithms in agent-based manufacturing scheduling systems," Integr. Comput.-Aided Eng., vol. 9, no. 3, pp. 207-218, 2002.

[97] W. Shen, Y. Li, H. Ghenniwa, and C. Wang, "Adaptive negotiation for agent-based grid computing," in Proc. AAMAS 2002 Workshop on Agentcities: Challenges in Open Agent Environments, Bologna, Italy, 2002, pp. 32-36.

[98] W. Shen and H. Ghenniwa, "Editorial of the special issue on agent-based manufacturing process planning and scheduling," J. Intell. Manuf., vol. 14, no. 5, pp. 427-428, 2003.

[99] W. Shih and K. Srihari, "Distributed artificial intelligence in manufacturing systems control," Comput. Ind. Eng., vol. 29, no. 1-4, pp. 199-203, 1995.

[100] T. Shintani and T. Ito, "An architecture for multi-agent negotiation using private preferences in a meeting scheduler," in Proc.5th PRICAI' 98, 1998, pp. 47-60.

[101] R. Sikora and M. J. Shaw, "Coordination mechanisms for multiagent manufacturing systems: Applications to integrated manufacturing scheduling," IEEE Trans. Eng. Manage., vol. 44, no. 2, pp. 175-187, May 1997.

[102] A. Sluga, P. Butala, and G. Bervar, "A multi-agent approach to process planning and fabrication in distributed manufacturing," Comput. Ind. Eng., vol. 35, no. 3-4, pp. 455-460, 1998.

[103] C. S. Smith and P. K. Right, "CyberCut: A world wide web based design-to-fabrication tool," J. Manuf. Syst., vol. 15, no. 6, pp. 432-442, 1996.

[104] R. G. Smith, "The contract net protocol: High-level communication and control in a distributed problem solver," IEEE Trans. Comput., vol. C-29, no. 12 , pp. $1104-1113,1980$.

[105] D. N. Sormaz and B. Khoshnevis, "Process planning knowledge representation using an object-oriented data model," Int. J. Comput. Integr. Manuf., vol. 10, no. 1-4, pp. 92-104, 1997.

[106] P. Sousa and C. Ramos, "A dynamic scheduling holon for manufacturing orders," J. Intell. Manuf., vol. 9, no. 2, pp. 107-112, 1997.

[107] P. Sousa, C. Ramos, and J. Neves, "The Fabricare scheduling prototype suite: Agent interaction and knowledge base," J. Intell. Manuf., vol. 14, no. 5, pp. 441-456, 2003.

[108] J. A. Stori and P. K. Wright, "A knowledge-based system for machining operation planning in feature based, open-architecture manufacturing," presented at the ASME Design Technical Conf., Irvine, CA, 1996.

[109] N. Sugimura, R. Hino, and T. Moriwaki, "Integrated process planning and scheduling in holonic manufacturing systems," in Proc. IEEE ISATP2001-Assembly and Disassembly in the 21 st Century, May 28-29, 2001, pp. 250-255.

[110] J. Sun, Y. F. Zhang, and A. Y. C. Nee, "A distributed multi-agent environment for product design and manufacturing planning," Int. J. Prod. Res., vol. 39, no. 4, pp. 625-645, 2001.
[111] K. P. Sycara, S. F. Oth, N. Sadeh, and M. S. Fox, "Resource allocation in distributed factory scheduling," in Intelligent Scheduling, M. Zweben and M. S. Fox, Eds. San Francisco, CA: Morgan Kaufmann, 1991, pp. 29-40.

[112] W. Tan, "Integration of process planning and scheduling-A review," $J$. Intell. Manuf., vol. 11, pp. 51-63, 2000.

[113] D. Trentesaux, C. Tahon, and P. Ladet, "Hybrid production control approach for JIT scheduling," Artif. Intell. Eng., vol. 12, no. 1, pp. 49-67, 1998.

[114] Y. J. Tseng and S. B. Joshi, "Recognizing multiple interpretations of interacting machining features," Comput.-Aided Des., vol. 26, no. 9, pp. 667688, 1994.

[115] Y. Tu, X. Chu, and W. Yang, "Computer-aided process planning in virtual one-of-a-kind production," Comput. Ind., vol. 41, pp. 99-110, 2000.

[116] J. M. Usher, "Negotiation-based routing in job shops via collaborative agents," J. Intell. Manuf., vol. 14, no. 5, pp. 485-499, 2003.

[117] H. V. Brussel, J. Wyns, P. Valckenaers, L. Bongaerts, and P. Peeters, "Reference architecture for holonic manufacturing systems: PROSA," Comput. Ind., vol. 37, no. 3, pp. 255-274, 1998.

[118] G. C. Vosniakos and B. J. Davies, "Knowledge-based selection and sequencing of hole-making operations for prismatic parts," Int. J. Adv. Manuf. Technol., vol. 8, pp. 9-16, 1993.

[119] L. Wang and D. H. Norrie, "Process planning and control in a holonic manufacturing environment," J. Appl. Syst. Stud., vol. 2, no. 1, pp. 106126, 2001.

[120] L. Wang and W. Shen, "DPP: An agent-based approach for distributed process planning," J. Intell. Manuf., vol. 14, no. 5, pp. 429-440, 2003.

[121] L. Wang, Q. Hao, and W. Shen, "Function block based integration of process planning, scheduling and execution for RMS," presented at the CIRP 2nd Int. Conf. Reconfigurable Manufacturing, Ann Arbor, MI, 2003.

[122] C. Wang, W. Shen, and H. Ghenniwa, "An adaptive negotiation framework for agent based dynamic manufacturing scheduling," in Proc. IEEE SMC, vol. 2, Washington, DC, Oct. 5-8, 2003, pp. 1211-1216.

[123] L. C. Wang and S. Y. Wu, "Modelling with colored timed object-oriented petri nets for automated manufacturing systems," Comput. Ind. Eng., vol. 34, no. 2, pp. 463-480, 1998.

[124] Y. H. Wang, C. W. Yin, and Y. Zhang, "A multi-agent and distributed ruler based approach to production scheduling of agile manufacturing systems," Int. J. Comput. Integ. Manuf., vol. 16, no. 2, pp. 81-92, 2003.

[125] R. R. Wu and H. M. Zhang, "Object-oriented and fuzzy-set-based approach for setup planning," Int.J. Adv. Manuf. Technol., vol. 14, pp. 406411, 1998.

[126] Y. Xu, L. Korba, L. Wang, Q. Hao, W. Shen, and S. Lang, "A security framework for collaborative distributed industrial control," presented at the IEEE Int. Conf. Industrial Informatics (CD-ROM), Banff, AB, Canada, 2003.

[127] Y. Yan, T. Kuphal, and J. Bode, "Application of multi-agent systems in project management," in Working Notes of the Agent-Based Manufacturing Workshop, Minneapolis, MN, 1998, pp. 160-170.

[128] B. P.-C. Yen and O. Q. Wu, "Internet scheduling environment with market driven agents," IEEE Trans. Syst., Man, Cybern. A, Syst., Humans, vol. 34, no. 2, pp. 281-289, Mar. 2004.

[129] S. H. Yeo, B. K. A. Ngoi, and H. Chen, "Process sequence optimization based on a new cost-tolerance model," J. Intell. Manuf., vol. 9, pp. 29-37, 1998.

[130] H. C. Zhang and E. Merchant, "IPPM-A prototype to integrate process planning and job shop scheduling functions," CIRP Ann., vol. 42, no. 1, pp. 513-518, 1993.

[131] F. Zhang, Y. F. Zhang, and A. Y. C. Nee, "Using genetic algorithms in process planning for job shop machining," IEEE Trans. Evol. Comput., vol. 1, no. 4, pp. 278-289, Nov. 1997.

[132] Y. Zhang, S. C. Feng, X. Wang, W. Tian, and R. Wu, "Object oriented manufacturing resource modeling for adaptive process planning," Int. J. Prod. Res., vol. 37, no. 18, pp. 4179-4195, 1999.

[133] F. L. Zhao and P. S. Y. Wu, "A cooperative framework for process planning," Int. J. Comput. Integr. Manuf., vol. 12, no. 2, pp. 168-178, 1999.

[134] F. L. Zhao, S. K. Tso, and P. S. Y. Wu, "A cooperative agent modeling approach for process planning," Comput. Ind., vol. 41, no. 1, pp. 83-97, 2000.

[135] W. H. M. Zijm, "The integration of process planning and shop floor scheduling in small batch part manufacturing," Ann. CIRP, vol. 44, no. 1, pp. 429-432, 1995.

[136] M. Zweben and M. S. Fox, Intelligent Scheduling. San Francisco, CA: Morgan Kaufmann, 1994. 


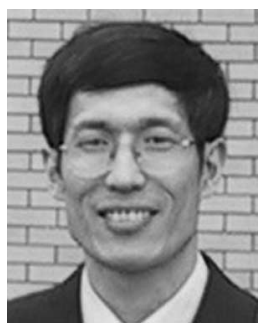

Weiming Shen (M'98-SM'02) received the B.Sc. and M.Sc. degrees in mechanical engineering from the Northern Jiaotong University, Beijing, China, in 1983 and 1986, respectively, and the Ph.D. degree in systems control from the University of Technologies of Compiegne, Compiegne, France, in 1996.

From 1986 to 1992, he was a Lecturer in mechanical engineering at the Northern Jiaotong University. $\mathrm{He}$ has also held positions with MediReport, Paris, France, and the Department of Mechanical and Manufacturing Engineering, University of Calgary. He is currently a Senior Research Scientist at the Integrated Manufacturing Technologies Institute, National Research Council Canada, London, ON. He is an Adjunct Professor in systems design engineering at the University of Waterloo and an Adjunct Professor in software engineering at the University of Western Ontario. He has authored more than 180 papers in scientific journals and international conferences/workshops, and coedited 11 conference/workshop proceedings in the related areas. He is also the author of Multi-Agent Systems for Concurrent Intelligent Design and Manufacturing.

Dr. Shen is a Member of ASME, ACM, AAAI, and CSCSI. He is a Registered Professional Engineer in Ontario.

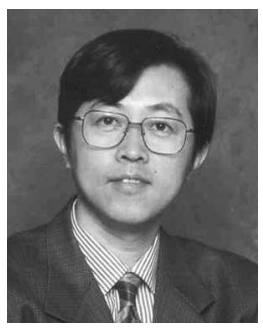

Lihui Wang received the B.Sc. degree in machine design from the Beijing University of Printing, Beijing, China, in 1982, and the M.Sc. degree in mechanical engineering and the Ph.D. degree in intelligence science from Kobe University, Kobe, Japan, in 1990 and 1993, respectively.

He has worked for two years at Kobe University and for two years at the Toyohashi University of Technology, Japan, as an Assistant Professor. Currently, he is a Senior Research Scientist at the Integrated Manufacturing Technologies Institute, National Research Council Canada, London, ON. He is also an Adjunct Professor at the University of Western Ontario, London, ON. He has published more than 100 papers in refereed journals and conference proceedings, and has edited four conference proceedings in manufacturing research.

Dr. Wang is a Senior Member of SME and a Member of the Japan Society for Precision Engineering. He is a Registered Professional Engineer in Ontario.

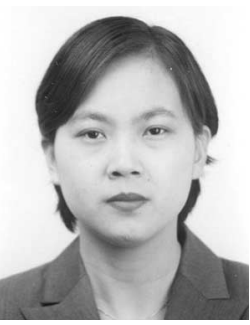

Qi Hao received the B.Sc. degree in mechanical design and manufacturing, the M.Sc. degree in electromechanical control and automation, and the Ph.D. degree in aeronautical and astronautical manufacturing engineering from the Northwestern Polytechnical University, Shannxi, China, in 1992, 1994, and 1997, respectively.

From 1998 to 2001, she was an Associate Professor at the Northwestern Polytechnical University. She has also held positions with Xi'an Butone, Inc. She is currently a Visiting Research Fellow at the Integrated Manufacturing Technologies Institute, National Research Council Canada, London, ON. She has authored or coauthored more than 30 journal and conference publications. Her research interests include manufacturing process planning and scheduling, ERP/MRP, virtual enterprises, supply chain management, and applications of intelligent agents and Internet/Web-based technologies to these areas. 